\title{
TETRATIC PHASE IN THE PLANAR HARD SQUARE SYSTEM?
}

\author{
K. W. WOJCIECHOWSKI ${ }^{1 *}$ AND D. FRENKEL ${ }^{2}$ \\ Institute of Molecular Physics, Polish Academy of Sciences \\ Smoluchowskiego 17/19, 60-179 Poznań, Poland \\ FOM - Institute for Atomic and Molecular Physics \\ Postbus 41883, 1009 DB Amsterdam, The Netherlands
}

(Rec. 4 December 2004)

\begin{abstract}
System of hard squares in two dimensions (2D) has been studied by Monte Carlo simulations. The simulations indicate that the isotropic fluid phase in this system does not freeze into a $2 \mathrm{D}$ 'crystalline' phase (of square lattice and quasi-long-range translational order) but transforms into an intermediate phase with the quadratic quasi-long-range orientational order (of coupled molecular axes and intermolecular bonds) and the translational order decaying faster than algebraically. The equation of state and the specific heat of the system are surprisingly well reproduced by smoothed version of the free volume theory in the whole density range.
\end{abstract}

Key words: melting in two dimensions, liquid crystals, hard convex body, equation of state, quasi-long range order

\section{INTRODUCTION}

Studies of simple, well defined models are the key to understand complex behaviours observed in real systems. A well known example of this approach is the hard sphere system which has been used for modelling liquids and their freezing [1]. Existing in nature liquid crystalline and plastic crystalline states, exhibiting intermediate order between crystals and liquids stimulated development of models with anisotropic hard molecules. It has been shown by computer simulations that purely geometrical interactions can lead to various phases whose existence is related to molecular anisotropy. Amongst them are various kinds of liquid crystals [2-4] and plastic crystals [2, 5, 6], which have been known in nature for long time. Recently, there were also found examples of new kinds of order, like the cubatic phase in the system of cut spheres [7, 8] or the degenerate crystal of hard dimmers [9, 10]. New phases have been shown to exist in systems of multi-rod molecules of zero volume [7]. Such molecules, being generalization of the Onsager's rods [11], allow for modelling generalized nematics, i.e. fluids with orientational order of any point group symmetry with an inversion centre [12]. Because the volume of real molecules is larger than zero, in contrast to the model multi-rods, a question arises if exotic, i.e. non-axial, nematics can be obtained in systems of hard body molecules of positive volume (and positive second virial coefficient). Cut spheres suggest

\footnotetext{
* Corresponding author: e-mail: kww@rose.man.poznan.pl
} 
positive answer to this question in three dimensions (3D). In two dimensions (2D) the answer to such a question is not known and it is one of problems addressed in the present paper.

Very simple 2D molecules, one can consider as candidates for mesogens of the exotic nematics, are the regular polygons. Such bodies represent special cases of convex bodies for which many general results has been obtained [13]; in particular the second virial coefficient is known exactly [14]. Amongst the regular polygons, these of a small number of sides (triangle, square, etc), the most different from the circle, seem to offer best chances to generate mesophases of symmetry different from the axial one. In the present paper we consider the square. In such a case the symmetry of the hypothetical exotic liquid crystalline phase should be the same as the symmetry of the molecule. (In the case of triangles one should expect hexagonal symmetry of a liquid crystalline phase, if such a phase would be stable.) The square 'molecule' is a limiting case of hard rectangular bodies for which some higher virial coefficients have been recently obtained [15] and whose phase diagram has been studied recently [16].

Hard squares are interesting not only as a potential mesogen for liquid crystalline phases. Studies of anisotropic particles in 2D can, in general, throw a new light on still controversial problem of 2D melting [17, 18] and possible kinds of order in 2D. Difference of topology between three-dimensional (3D) and two-dimensional (2D) systems allows for qualitative differences in the nature of order and melting transition in three and two dimensions. 2D "crystals" with short range interactions between molecules cannot exhibit any true long-range translational order at positive temperature [19-22] because the long-wave phonons lead to logarithmic increase of positional fluctuations of the molecules. The decay of translational correlations in $2 \mathrm{D}$ has a power law form and leads to ordering known as quasi-long range order. (In one dimension the fluctuations are proportional to the size of the system and, hence, destroy any crystalline order completely. The correlations decay in the exponential way, what is typical for fluids.) This is in contrast with crystals in 3D for which periodicity, implied by the long range translational order, is one of the most fundamental properties. The periodicity of $3 \mathrm{D}$ crystals is lost only at melting, and the melting itself is, according to the general experience, a first-order phase transition. The lack of a true long-range translational order in 2D crystals does not imply, however, as one might conclude from the theory proposed by Kosterlitz and Touless [23], that the melting transition between a low temperature (high density) 2D solid and a high temperature (low density) fluid is continuous. Computer simulations strongly indicated first order character of melting in 2D model systems with short range interactions (for references see the review papers in Refs. [24-27]). Barker and Henderson calculated explicitly the positional fluctuations in the hard disc system and concluded that they are quantitatively very small even for systems of macroscopic size [24]. Such observations might suggest that the nature of melting of 2D systems with "physical", i.e. short range, interactions is not much different from that observed in 3D systems. Simulations of large Lennard-Jones system [28] showed, however, that it is not so. No two-phase coexistence in the standard meaning was found in the melting region in this system. On the contrary, the 
system appeared to be quite homogeneous in this region [28, 29]. Moreover, in the intermediate region between fluid and 'crystal', a quasi-long-range order of bonds connecting the nearest neighbouring particles has been found [28].

It is generally accepted that in the absence of the translational long-range order, the longrange orientational order of bonds connecting the nearest neighbouring particles is that which is lost at melting of $2 \mathrm{D}$ crystals. In the theory of Kosterlitz and Thouless [23] the process responsible for destruction of this order is the dislocations unbinding [23]. Halperin and Nelson [30] noticed that dislocation unbinding alone cannot lead to isotropic fluid. They proposed a second transition, dislocations unbinding, as the final step in the $2 \mathrm{D}$ melting. According to their theory, generalized by Young [31] (and further referred to as the KTHNY theory), a 2D crystal may melt via two second-order transitions. In such a case the 2D crystal is separated from the fluid by a new phase with quasi-long-range orientational order and without translational order. Computer simulations did not support such a scenario for isotropic particles with short-range interactions [25-27]. On the other hand, some experiments performed with liquid crystalline layers indicated relevance of the two-stage melting to such systems [32]. Kleinert proposed a certain lattice model explaining qualitatively this situation [33]. In the free energy expansion he considered an additional term which he related to molecular anisotropy [33]. Janke and Kleinert [34] studied this model and observed either a single first-order transition or two continuous transitions, depending on the value of a coefficient at the new term. It is not obvious, however, if and when the expansion proposed by Kleinert is applicable to real systems. Although his results indicate that molecular anisotropy is required for two-stage melting, they cannot be seen as a definite argument that the molecular anisotropy is sufficient for such a melting scheme. Hence, a question arises if the molecular anisotropy itself can lead to a two-stage melting in two dimensional systems and, if yes, how large should it (the molecular anisotropy) be.

In general, translational-rotational coupling present in systems of anisotropic particles may lead to qualitatively new orderings, absent in the case of isotropic particles. In the case of square particles one cannot exclude a priori neither an orientationally disordered (plastic) crystal nor orientationally ordered (liquid crystalline) fluid. In the latter case the molecular anisotropy may, in principle, lead to any of three possibilities: (i) a phase with molecular orientational (MO) order and without bond orientational (BO) order (nematic phase of 4-fold symmetry), (ii) a phase with a BO order and without a MO order, and - if the molecular orientations couple to the bond-bond orientations - (iii) a phase (further referred to as tetratic) with both (4-fold) MO and BO order. The first two cases, with no coupling between molecular orientations and bonds do not seem to be plausible in the case of hard squares, if one takes into account results of the Refs. [35, 36]: systems with non-separable interaction potential are expected to exhibit a coupling between the molecular orientations and the bond orientations. At present the most efficient way to check if any of the possibilities mentioned above holds in the case of the squares are Monte Carlo simulations. 
One more reason for which the squares constitute theoretically attractive system is a strong degeneracy of their close packed structure. Squares can be densely packed into uncorrelated parallel rows what may allow for non-crystalline order even at highest densities; compare the case of hard dumb-bells of high anisotropy [9, 10]. In the case of squares, in place of the 2D crystalline phase, one might find a thermodynamically stable $2 \mathrm{D}$ analogon of a $3 \mathrm{D}$ smectic or columnar phase.

Although the hard square 'molecule' seems to be a rather odd object, both for its idealized shape and the 2D nature, the remarks presented above encouraged us to its study. Discussing the problem of the shape, we would like to add here that square shape of a molecule is expected to favour structures of square symmetry which do not need to be elastically isotropic. This is a complication from one side and a chance for new behaviours from the other. The problem of physical meaning of 2D models is also worth to be mentioned. Although one could object that 2D systems are not physical at all, as real systems are composed of atoms and molecules which have dimensionality three, 2D models are interesting for studies of influence of the dimensionality on various properties of many body systems. Except the purely theoretical significance, 2D models are also convenient idealizations of real systems such as, e.g., thin layers and films adsorbed on solid surfaces or on surfaces of liquids. One should stress that, in the case of liquid crystals, thin layers and films are of great importance for practical applications.

The structure of the paper is as follows. Brief description of the simulation method used is contained in the section 2. In the section 3 the simulation results of the equation of state (EOS) and the specific heat are compared with some theoretical approximations. Studies of the structure and the order in the system are presented in the section 4. Section 5 contains the summary and conclusions.

\section{THE SIMULATIONS}

Simulations were performed using Monte Carlo (MC) method in the constant pressure (NpT) ensemble. Two sizes of the system were studied thoroughly: $N=196$ particles, further referred to as the small $(S)$ system and $N=784$ particles, further referred to as the large $(L)$ system. At certain values of pressure, characteristic for different structures observed in the system and covering the transitions between them, some runs were also performed for the largest $(X L)$ system of $N=3136$ particles. The particle moves consisted of simultaneous translations and orientational changes. The maximum amplitudes of the $x$ - and $y$-components of the translational displacements were equal to the maximal amplitude of the change of particle orientation (measured in radians). The acceptance ratio for the particle moves was between 30 and 40 percent.

In the case of the $S$ system the typical length of a run used to calculate the EOS was $2 \times 10^{4}$ cycles after equilibration of the same length. For the $L$ system the runs, in which the averages were computed, were twice as long. Final configuration of a previous run in a se- 
quence was used as the starting configuration in the following run of the sequence. Box moves during equilibration were tried $N^{1 / 2}$ more often than single particle moves. After equilibration, the number of trial moves of the box was equal to the number of the trial moves of a single particle (i.e. one per cycle). The acceptance ratio for the box moves was between 20 and 30 percent. In the case of runs performed in the fluid phase the shape of the box of periodicity was typically fixed as a square, whereas for the solid phase the box was a rectangle of variable shape. In the transition region, as well as to calculate the orientational correlations in the system, extra runs, five or more times longer than those used in the EOS computations, were performed.

For the $X L$ system the initial configurations were either perfect crystalline lattices (of the density estimated from the isotherm of the $L$ system) or uniformly expanded equilibrium configurations obtained at higher pressures or configurations obtained by quadrupling the $L$ system. Typical runs were $(1 \div 2) \times 10^{5}$ cycles long with one trial move of the box per cycle.

\section{THE EQUATION OF STATE AND THE SPECIFIC HEAT}

\subsection{The EOS}

For $N=196$ squares two series of runs were performed. In the first series, the particles were moved in a rectangular box of variable shape, and the pressure was decreased in

Fig. 1. (a) The initial structure

a

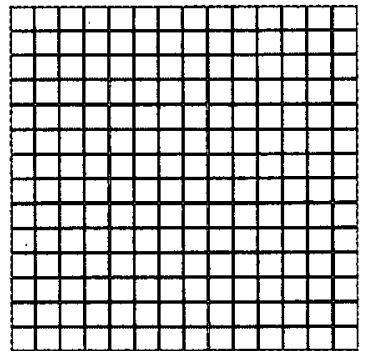

for the expansion experiment of the $S$ system, (b)-(d) Snapshot configurations of the $S$ system at $p=12, p=9$, and $p=7$, respectively b
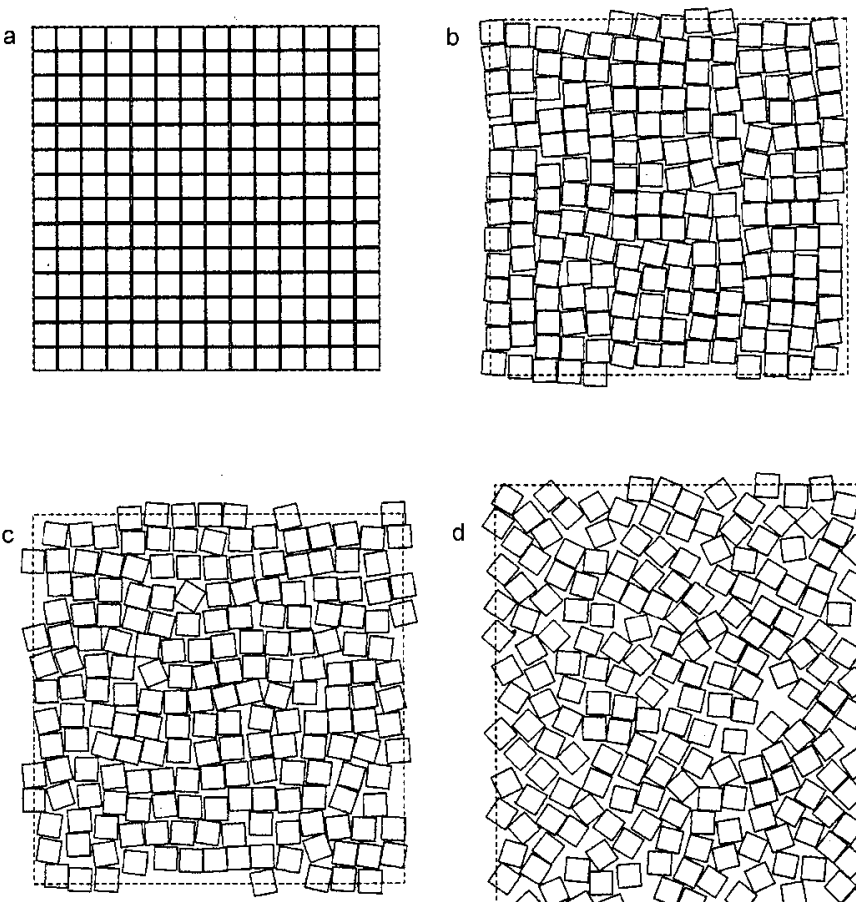

d

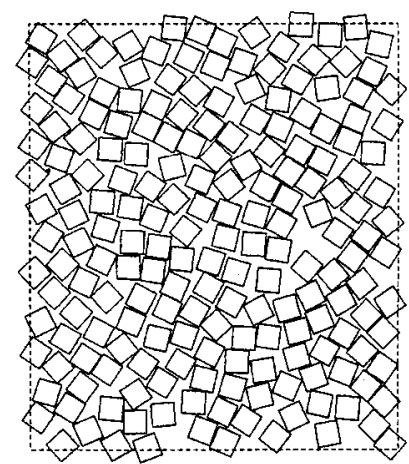


subsequent runs from $p=30$ down to $p=3$. The initial structure at $p=30$ was a perfect $14 \times 14$ square lattice with particle sides oriented parallelly to the sides of the box, see Fig. la. The computed density was a smooth function of the pressure down to $p \approx 8$ where a cusp was observed in the isotherm. The second series, performed in the square box, was started at $p=0.5$ and the pressure was increased up to $p=10$. No hysteresis was observed around the cusp near $p=8$; this may suggest continuous nature of the melting transition in the system; the isotherms are shown in Fig. 2a. Some snapshot configurations of the squares at various pressures are shown in Fig. 1b-d.
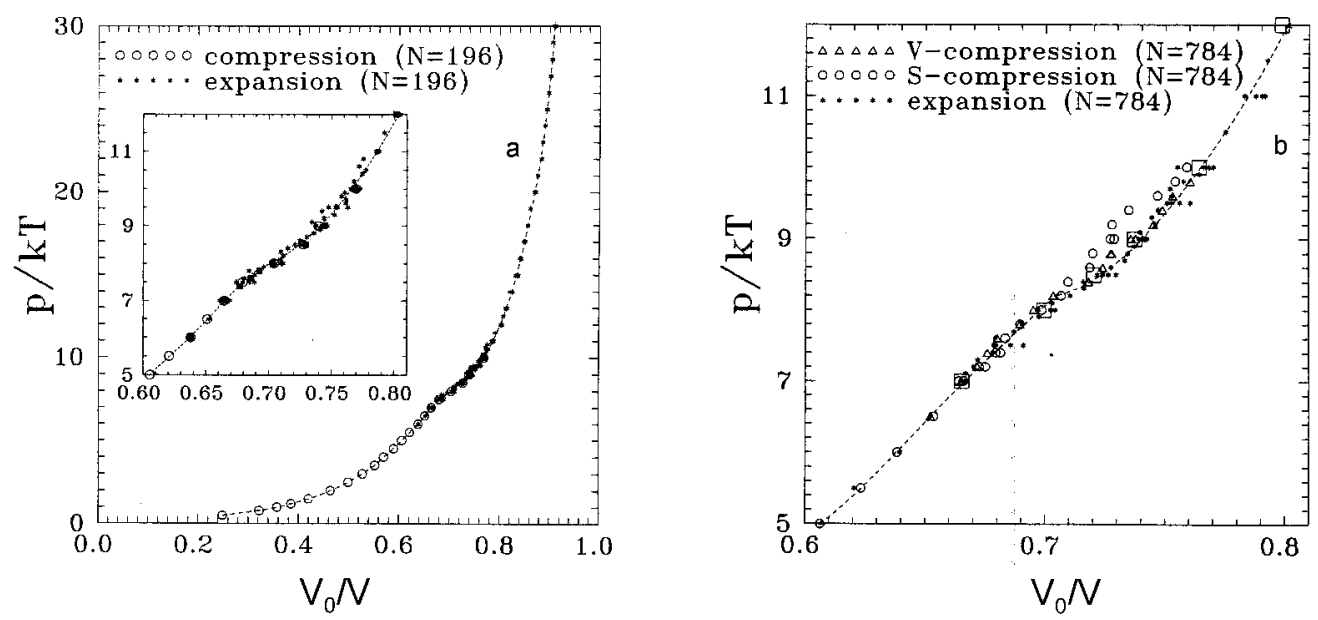

Fig. 2. it (a) The isotherm of the $S$ system. Circles represent results of the series with increasing pressure in subsequent runs, and square box of periodicity. Stars correspond to the series with decreasing pressure and rectangular box of variable shape. The lines are drawn to guide the eyes. The full squares in the insert represent the longest runs whose results are collected in Table 1. it (b) The isotherm of the $L$ system. Circles, stars and lines have the same meaning as in (a). The triangles correspond to the series with increasing pressure and a box of variable shape. The open squares represent the runs tabulated in Table 1

To check the dependence of the EOS on the system size, the experiment was performed in the same way for $N=784$ squares. In the case of expansion no differences exceeding the experimental error were noticed between the isotherms of the $S$ system and the $L$ system, except in the very neighbourhood of the cusp, where the isotherm of the $L$ system was located slightly above that of the $S$ system. The same was true for the cusp itself. Below the cusp the series with increasing pressure did not show any density differences, when compared with that with decreasing pressure. However, rather large differences were obtained above the cusp: the densities in the compressed $L$ system were about 2 percent lower than in the system under expansion. The EOS branch corresponding to the compression experiment was smooth and did 
not exhibit any cusp-like singularities near $p=8$, see Fig. 2b. Such a behaviour, typical for first-order melting, is completely different than that obtained for the $S$ system.

Table 1. The equilibrium densities, $\rho_{z}$ (the subscript $Z=S, L, X L$ denotes the size of the sample), in the hard square system obtained for very long runs $\left(0.1 \div 1 \times 10^{6}\right.$ cycles $)$ at a few pressures and system sizes; the experimental errors for the density do not exceed 2 per mile

\begin{tabular}{cccc}
\hline$p^{*}$ & $\rho_{S}$ & $\rho_{L}$ & $\rho_{X L}$ \\
\hline 20 & .8715 & - & .8712 \\
15 & .8362 & .8390 & - \\
12 & .8012 & .7984 & .8009 \\
10 & .7689 & .7640 & .7588 \\
9 & .7444 & .7371 & .7310 \\
8.5 & .7294 & .7201 & .7155 \\
8 & .7030 & .6994 & .6961 \\
7.8 & .6926 & - & - \\
7.6 & .6857 & - & - \\
7.4 & .6772 & - & - \\
7 & .6660 & .6653 & - \\
6 & .6379 & - & - \\
4 & .5725 & - & - \\
2.5 & .5027 & - & - \\
\hline
\end{tabular}

Analysis of snapshot configurations of the compressed $L$ system revealed existence of some crystalline grains of different orientations for pressures above the cusp obtained in the expansion experiment. At higher pressures the grains have disappeared. Some vacancies and dislocations remained, however, 'frozen in' into the system because the square boundary conditions were inconsistent with the sample orientation. To remove this obstacle we performed another series of runs with increasing pressure. This time the periodic box was a parallelogram of variable shape. The resulting isotherm showed a cusp at the same pressure as obtained in the expansion experiment. No differences exceeding the experimental error were observed this time between the expansion and compression results, see Fig. 2b. Such a situation suggests that the 'compression' branch of the EOS obtained in the square box is only metastable, and the transition occurs rather smoothly near $p=8$.

A few runs were also performed for the $X L$ system. We observed further decrease of densities for pressures in the vicinity of the cusp. The comparison of the EOS data obtained from long runs for some pressures at various $N$ is presented in Table 1 . It can be seen there that the system exhibits large number dependence of the EOS in the intermediate region. Outside this region we did not observe any number dependence of the EOS exceeding the experimental error: for $p \leq 7$ and $p>12$ the obtained densities were, within the accuracy 
of the simulations, the same as in the $L$ and $S$ systems. The number of the data points in the case of $X L$ system is, clearly, not sufficient to distinguish, by analysis of the EOS only, between a discontinuous (weakly first-order) - and a continuous (cusp-like) transition.

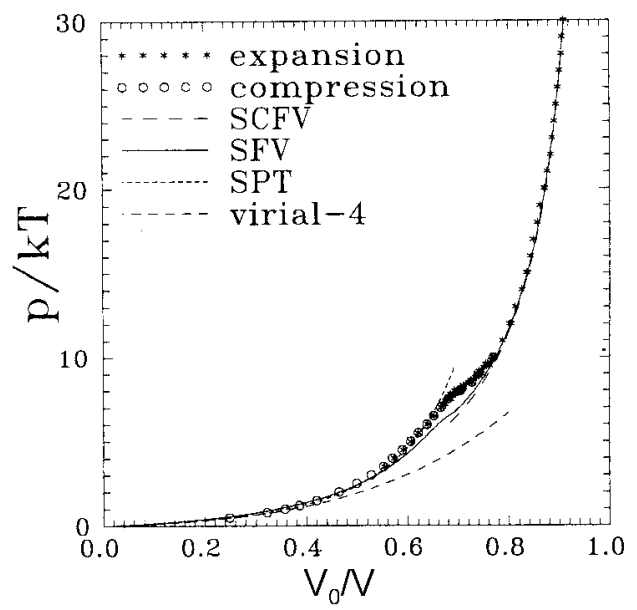

Fig. 3. Comparison of the Monte Carlo results at $N=196$ with some theoretical approximations: the self-consistent free volume theory (SCSF), the smoothed free volume approximation (SFV), the scaled particle theory (SPT), and the virial expansion truncated at the fourth term

In Fig. 3 the experimental results are compared with some theoretical approximations. At low densities these are: (i) the fourth-order virial expansion:

$$
p^{*}=\rho+B_{2} \rho^{2}+B_{3} \rho^{\hat{3}}+B_{4} \rho^{4}
$$

and (ii) the scaled particle theory (SPT) [14]:

$$
p^{*}=\frac{\rho\left(1+B_{2} \rho\right)}{(1-\rho)^{2}}
$$

where $p^{*}=p / k_{B} T, B_{2}=1+4 / \pi[14]$ and the virial coefficients $B_{3}$ and $B_{4}$ are taken from the Ref. [15]. It can be seen that the virial expansion considerably underestimates the MC data for $\rho>0.25$. Much better, although still not perfect, agreement is offered by the SPT.

At high densities the results of the self-consistent free volume approximation (SCFV) are shown. The SCFV is a kind of a one-particle mean field treatment [37]), which can be solved exactly in the case of squares [38]. As it can be seen the agreement between the MC data and the SCFV is quite good for $p>11$.

It is interesting to notice that a smoothed version of the free volume (SFV) theory (obtained by assuming that each molecule moves in a cell formed by lines tangent to its nearest neighbours fixed at their lattice positions and orientations) reproduces very well the shape of the isotherm in the whole density range. The EOS obtained within this approximation exhibits a cusp at $\rho=(3 / 4+1 / \sqrt{2})^{-1} \approx 0.68629$, i.e. at the value above which the molecules 
can rotate in the cells. Comparison with the MC data may suggest that the cusp in the actual isotherm is related to a rapid change of the orientational order in the system. We will return to this question in the next section.

\subsection{The specific heat}

The EOS of a hard body system is closely related to its constant-pressure specific heat per particle, $c_{p}$ :

$$
c^{p}=\frac{d}{2} k_{B}+\frac{p^{* 2}}{\rho}\left(\frac{\partial \rho}{\partial p^{*}}\right) k_{B},
$$

where $p^{*}=p / k_{B} T$, and $d$ is the number of degrees of freedom of a particle, ( $d$ is equal to 3 for any stiff $2 \mathrm{D}$ hard anisotropic particles for which translational and rotational moves are allowed.) The first, constant term in the right-hand side of the above equation is equal to the specific heat per particle at constant volume, $c_{v}$. The second term could be obtained, in principle, by differentiating the EOS. Another, independent way to calculate this term is via computing the volume fluctuations which can be expressed as:

$$
\left\langle(\Delta V)^{2}\right\rangle=N k_{B} T \frac{1}{\rho^{2}}\left(\frac{\partial \rho}{\partial p^{*}}\right) .
$$

Fig. 4. Comparison of the constant pressure specific heat per particle obtained in the $S$ system of hard squares with the result of the SFV approximation

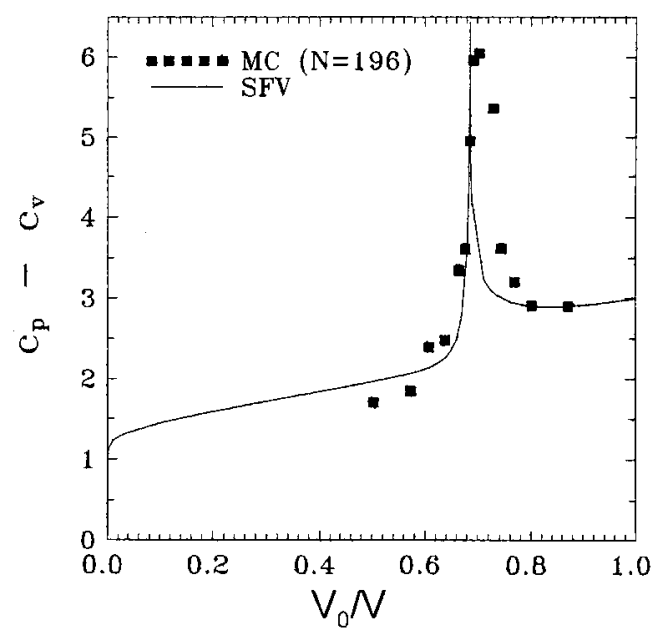

Computations of the volume fluctuations require, however, much longer runs than the EOS itself, and the length of the runs increases as the second power of the number of particles in the system. In the present studies only the longest runs of the $S$-system with the runs of 
$(0.5 \div 1) \times 10^{6}$ cycles were long enough to allow for a meaningful comparison with the smoothed free-volume theory, so successful in the case of the EOS. The comparison, presented in Fig. 4, shows that the SFV appears to be also a reasonable approximation for the specific heat of the system. The sharp peak in the SFV's specific heat, located at the same density where the cusp in the EOS is obtained within this approximation, indicates rapid release of the orientational degree of freedom of the molecules at the transition. Rough results obtained for the specific heat of the larger systems suggest that with increasing $N$ the peak moves to higher densities and its amplitude increases.

\subsection{Shape of the sample}

Simulations performed in the box of variable shape allow the system easily to reach the equilibrium structure. In Fig. 5 the pressure dependence of the sides of the periodic box is shown for the $S$ and $L$ systems. It can be seen that the box sides are (within the experimental error) equal down to $p \approx 10.5$, i.e. the box preserves its initial, square shape. Resistance against the shape changes is typical for solids, and we interpret the above behaviour as indication of solidity of the system at high pressures. The shape of the box suggests that a (locally) crystalline phase of square unit cell is stable in the system. For lower pressures the shape of the box fluctuates indicating decrease of the resistance of the system to changes of the shape. The system behaves like a fluid. It should be stressed that this fluid-like behaviour is observed much above the cusp of the EOS.

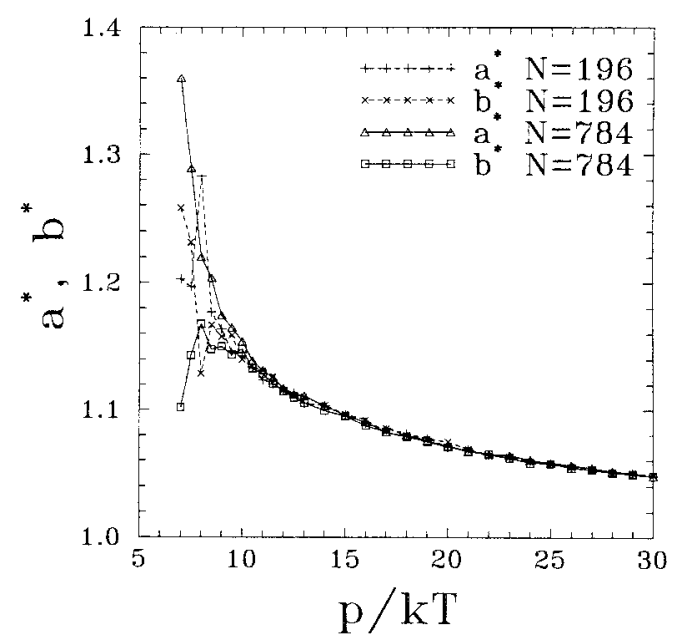

Fig. 5. Pressure dependence of the box sides (divided by their values at close packing) in the $S$ and $L$ system

The above results suggest that the EOS of the hard square system consists of (at least) two branches separated either by a continuous, e.g. cusp-like, transition or a weekly first order transition. Summarizing the results of the present section and anticipating some results of 
the next section, we will call the lower branch - fluid. In the upper branch we will, perhaps somehow arbitrary at the moment, distinguish two regions, above and below $p=11$. In the first of them $(p>11)$ the SCFV theory rather well approximates the isotherm of the system. As the SCFV approximation is known to reproduce well various dense solid phases [38], this region will be further referred to as a solid one. The remaining region, between the cusp and the solid, will be referred to as an intermediate region.

\section{STRUCTURE AND ORDER IN THE SYSTEM}

The structure and the order in a molecular system can be determined by analysis of ordering of molecular axes and the space distribution of molecular centres of mass. We will start with description of the orientational order of the squares.

\subsection{The orientational order of molecules}

A rough information on the orientational order can be obtained by analysis of the orientational singlet distribution function (OSDF) which is the probability that a molecule has a given orientation. The histogram of molecular orientations (modulo $\pi$ ), obtained at a few pressures in the $S$ system, is plotted in Fig. 6. The range of $\varphi$ in the histogram was two times larger than that required by the symmetry of the square. This allowed for observation of the orientational diffusion in the system. Down to $p=14$, what corresponds to $\rho \approx 0.82$, only librations of squares around their initial, $\varphi=0$, direction were observed. This corresponds to positive values of the OSDF near the single maximum at $\varphi=0$ and zero values elsewhere. At

Fig. 6. The OSDF function (normalized in such a way that it is equal to unity in the isotropic fluid) obtained in the $L$ system at a few pressures

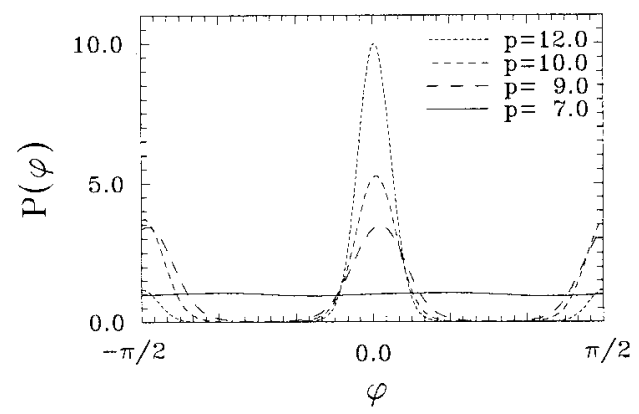

lower pressures a second maximum developed at $\varphi=\pi$, proving that the molecules started rotation. Very small values of the OSDF between the maxima indicated 'jump-rotations' of the squares down to $p \approx 8$, where a rapid increase of the OSDF's minimum was observed. At low pressures the OSDF is flat, i.e. no orientation is preferred. Values of the minima and maxima of the OSDF obtained from the histogram of the $S$ system are shown in Fig. 7a as a function of the pressure. In Fig. $7 \mathrm{~b}$ analogous plot is presented for the $L$ system. As it can be 
seen, the intermediate region, where the pressure dependences of the both quantities are steep, is slightly narrower in the $L$ system and shifted to higher pressures. One can also notice that the orientational order remaining in the fluid phase decreases with the increase of the system size.
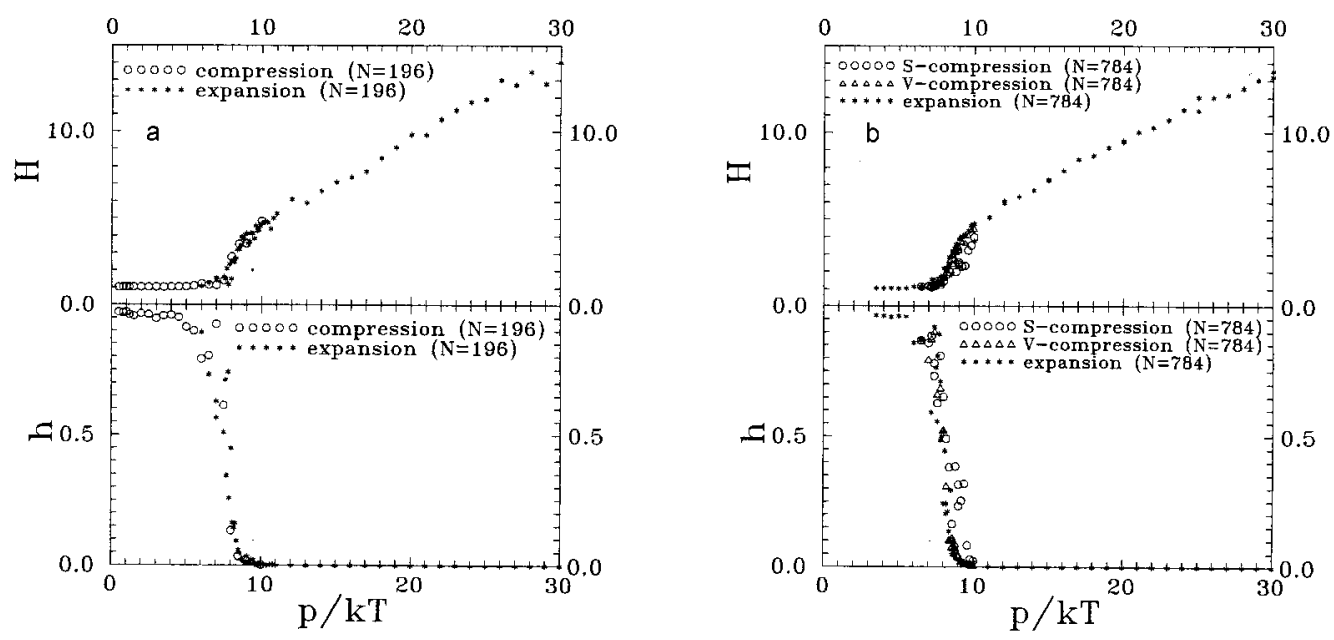

Fig. 7. The pressure dependence of the minimal and maximal values of the OSDF function: (a) $N=196$, (b) $N=784$

More detailed information on the orientational order of the molecules can be obtained from analysis of the pair correlation function of the molecular orientations, $g_{4}{ }^{(\mathrm{MO})}(r)$, defined as:

$$
g_{4}^{(\mathrm{MO})}(r)=\langle\cos [4 \varphi(r)-4 \varphi(0)]\rangle
$$

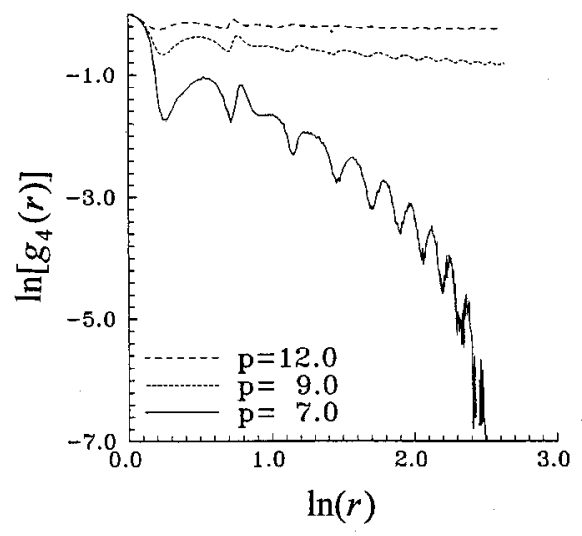

Fig. 8. Plots of the orientational correlation function, $g_{4}(r)$, in the $L$ system at three pressures characterizing the three regions in the phase diagram of the system: solid $(p=12)$, intermediate $(p=9)$, and fluid $(p=7)$ 
In Fig. $8 g_{4}(r)$ of the $L$ system (in the $S$ system the fast decay at $p=7$ is not so well visible for obvious reasons) is plotted in the $\log$ - log scale at three pressures representing the three regions distinguished in the EOS of the system. It can be seen that the (almost) flat curve in the solid region exhibits negative slope in the intermediate region and becomes negatively curved in the fluid. Assuming algebraic decay of the correlations:

$$
g_{4}(r) \sim r^{-\eta_{\varphi}}
$$
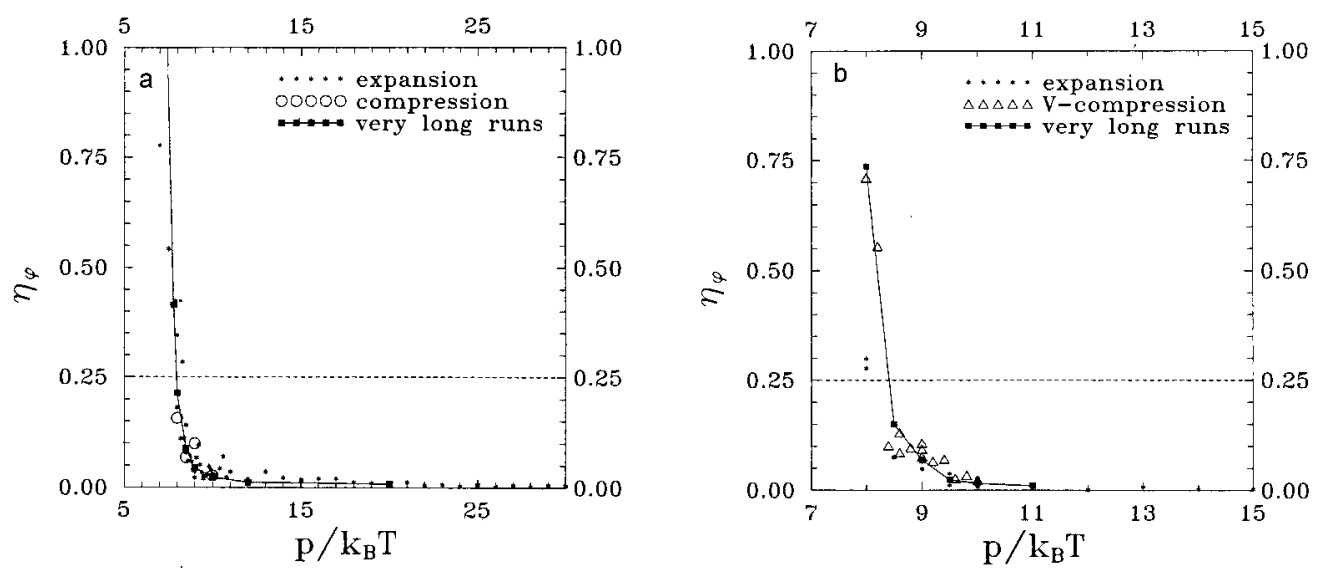

Fig. 9. Pressure dependence of the exponent $\eta_{\varphi}$ obtained by assuming the algebraic decay of the MO correlation function $g_{4}^{(\mathrm{Mo})}(r)$ :(a) $N=196$, (b) $N=784$. In the case (b) the triangles correspond to compression in the box of variable shape

one can calculate values of the exponent $\eta_{\varphi}$. In the present paper the exponents were obtained from linear fits to the correlation function plotted versus the distance in the log-log scale. (Obviously, the computed values of the exponent $\eta_{\varphi}$ are meaningful only when the plot does not exhibit any curvature.) In Fig. 9a the computed values are plotted for the $S$ system in a range of pressures. The points exhibit some scattering. It can be seen, however, that $\eta_{\varphi}$ is very close to zero in the solid and rapidly increases in the intermediate region. It is worth to notice that $\eta_{\varphi}$ is equal to $1 / 4$ in the vicinity of $p=7.9$, the pressure corresponding to the cusp in the EOS. ( $1 / 4$ is the value predicted by the Kosterlitz and Thouless theory for the exponent of the bond-bond correlations in isotropic systems.) In Fig. $9 \mathrm{~b} \eta_{\varphi}$ is plotted for the $L$ system. The value $1 / 4$ is reached between $p=8$ and $p=8.5$, i.e. again near the cusp in the EOS of the $L$ system. For the $X L$ system the value $1 / 4$ is reached above $p=8.5$. Unfortunately, we did not succeed to calculate the exponents $\eta_{\varphi}$ at $p=9$. The computed values differed significantly from one run to another. This suggests that fluctuations are so large that runs as long as $2 \times 10^{5}$ cycles are too short for this size of the system. These results show that the position of 
the point where $\eta_{\varphi}$ changes from very small values to $1 / 4$ strongly depends an the system size and this point moves to higher pressures and densities with the increasing size of the system.

In the fluid phase one expects short range orientational order of the molecular axes and the MO pair correlation function having the exponential form:

$$
g_{4}(r) \sim \exp \left(\frac{-r}{\xi_{4}}\right)
$$

The KTHNY theory predicts that $\xi_{4}$ diverges when the isotropic fluid reaches the temperature of the transition to the ordered phase. In a hard body system the temperature is proportional to the inverse pressure, and the KTHNY-dependence of $\xi_{4}$ near the transition temperature $T_{i}$ has the form:

$$
\xi_{4}=\exp \left(\frac{b}{\left|T-T_{i}\right|^{1 / 2}}\right)=\exp \left(\frac{b}{\left|1 / p^{*}-1 / p_{i}^{*}\right|^{1 / 2}}\right) .
$$

We calculated the values of $\xi_{4}$ at a few pressures and used them to determine the point where the disclinations unbind. This is illustrated in Fig. 10 for the $S$ system. It can be seen that the point where disclinations unbind coincides with the cusp in the EOS and the point where $\eta_{\varphi}=1 / 4$.

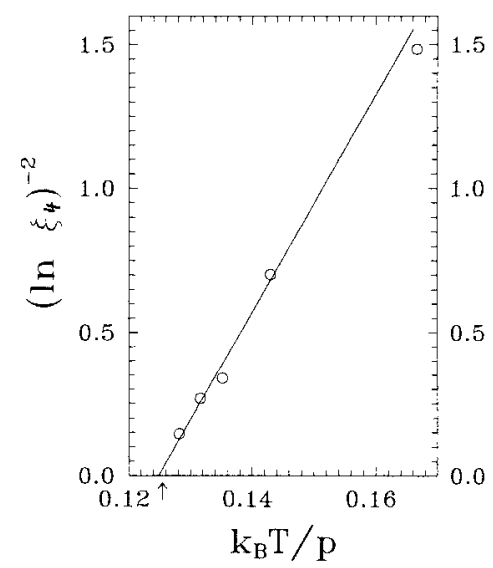

Fig. 10. The inverse-pressure dependence of the orientational correlation length $\xi_{4}$ in the $S$ system. The arrow indicates the point at which the exponent $\eta_{\varphi}$ reaches the value $1 / 4$

The ordering of the molecular centres can be characterized by the radial distribution function and by correlations of the bonds between the centres of nearest neighbours.

\subsection{The bond-bond order}

Computation of the bond-bond orientational correlation function (its definition is the same as that of $g_{4}^{(\mathrm{MO})}(r)$ except that molecular orientations are replaced by orientations of inter- 
particle bonds), $\quad g_{4}^{(\mathrm{BO})}(r), \quad$ requires much more computer time than the correlations of the molecular axes do. In the square system one can avoid the latter computations as the molecular axes are strongly coupled with the intermolecular bonds. This can be seen in Fig. 11, where exponents obtained from power fits to both functions are compared: the exponents are equal within the experimental error. It follows from this figure that the hard square system does not form a phase with only a single kind of the orientational order, i.e. neither a phase with MO and without $\mathrm{BO}$ order nor a phase with $\mathrm{BO}$ and without MO can exist in this system.

Fig. 11. Comparison of the MO-decay exponent, $\eta_{\mathrm{b}}$ $\eta_{\varphi}$ with the BO-decay exponent, $\eta_{b}$

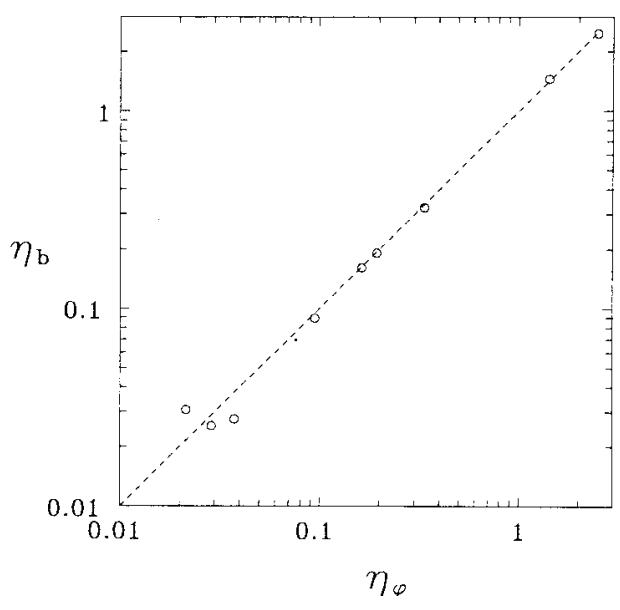

The existence of the strong coupling between the MO and the BO can be used to explain the presence of the sharp peak observed in the specific heat. Such a peak is not expected at the disclination unbinding which, according to the KTHNY theory, is responsible for the destruction of the BO order and which should be accompanied by a small bump in the specific heat [30]. The strong coupling between the MO and the BO implies that destruction of the BO order is accompanied by the destruction of the MO order. The latter is responsible for the strong peak in the specific heat, reproduced very well by the SFV approximation.

\subsection{The translational order of the molecular mass centres}

The radial distribution function in the solid region displays strong maxima of slowly decaying amplitude. At high pressures this function has a rather complicated and irregular structure. At lower pressures this function is similar to that shown in Fig. 12. It is easy to see that its decay is algebraic (Fig. 13a). We did not determine the value of the exponent of the algebraic decay of the radial distribution function at high pressures because its highly structured form makes this procedure very inaccurate. At lower pressures the exponent can be estimated more accurate, and its value at $p=12$, i.e. near the point where the radial distribution function changes the character of the decay of its maxima, appears to be close to 


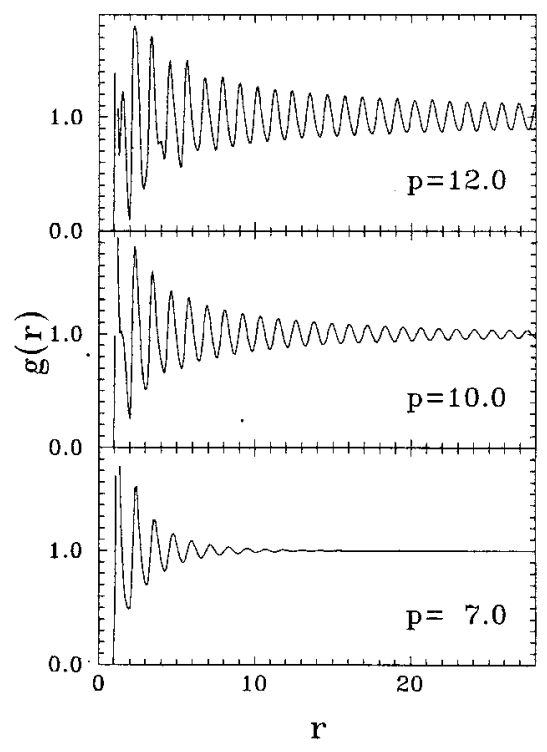

Fig. 12. Radial distribution function, $g(r)$, in the $X L$ system at three pressures representing different regions of the EOS
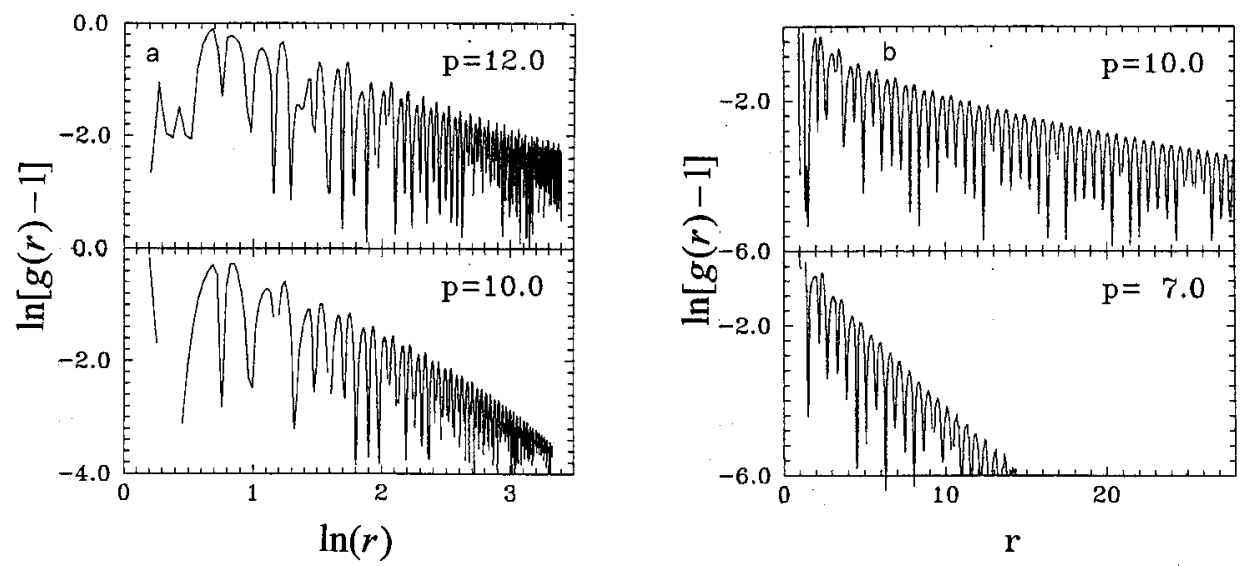

Fig. 13. (a) Log-log plot of the radial distribution function at $p=12$ and $p=10$. (b) Log- $r$ plot of the radial

function

at $p=$

10 and $p=$

7

$2 / 3$ what is twice as large as in the case of the isotropic system [30]. In the intermediate region the amplitude of the maxima of the radial distribution function decays also rather slowly (Fig. 12). The decay is, however, clearly faster than the algebraic one (Fig. 13a). At large distances the decay can be reasonably approximated by the exponential (Fig. 13b). In the fluid the amplitude of the maxima decays quickly (Fig. 12), and the decay is clearly exponential (Fig. 13b). Assuming the exponential decay:

$$
g(r)=\exp (r / \xi)
$$


in the intermediate region and in the fluid, we calculated the correlation length, $\xi$, in the $S$ system (see Table 2). Assuming that, when the solid phase is approached, the correlation length diverges in the similar way as it was predicted for the isotropic case [30]:

$$
\xi=\exp \left(C\left|T-T_{m}\right|^{-v}\right)=\exp \left(C\left|1 / p^{*}-1 / p_{m}^{*}\right|^{-v}\right)
$$

one could try to locate the transition between the crystal and the tetratic phase. Unfortunately, the exponent $v$ has not been determined in the case of the 4-fold symmetry. However, if we assume that $v$ is equal to $v_{\text {iso }}=0.369635$, what is value in an isotropic system [35], the transition should occur at $p_{m}=10.3$ what is close to the value at which increasing fluctuations of the box shape were observed (Fig. 5). Assuming that $v=0.25$ or $v=0.5$ the transition shifts to $p=9.8$ or $p=10.8$, respectively. For $v=v_{\text {iso }}$ one obtains $p_{m}=10.5$ in the $L$ system, and $p_{m}=10.4$ in the $X L$ system. Hence, location of the transition from the solid to the tetratic phase only weekly depends on the system size; it seems rather safe to expect that in the thermodynamic limit it occurs not far from $p=10.5$.

Table 2. The translational correlation length, $\xi_{z}$ (the subscript $Z=S, L, X L$ denotes the size of the sample), determined from the equation 10 in the studied samples of $N=196,784$, and 3136 squares

\begin{tabular}{cccc}
\hline$p^{*}$ & $\xi_{S}$ & $\xi_{L}$ & $\xi_{X L}$ \\
\hline 8 & 3.56 & 3.55 & 3.45 \\
8.5 & 5.8 & 5.35 & 5.4 \\
9 & 7.1 & 7.3 & - \\
10 & 10 & 10.2 & 11.6 \\
\hline
\end{tabular}

Strong configurational degeneracy of the close packing limit of squares allows for nonperiodic arrangements in this system even at very high densities. In the case of parallel squares of fixed orientation various versions of the free volume theory predict that a $2 \mathrm{D}$ analogue of a columnar or smectic phase is more stable than the square lattice [38]. When the squares can rotate the free volume theory is not so conclusive as its result depends essentially on the details of the approximation [38].

Simulations of the $S$ system showed sliding of columns and rows of squares even at the highest densities studied (Fig. 14a). The snap-shot configurations revealed some neighbouring rows and columns of the squares 'out of phase' (i.e. shifted by a distance different from the average distance of centres of the squares which could be interpreted as the lattice constant). Such results might be interpreted as a support for stability of the 'columnar' phase. However, one should be aware that sliding of rows/columns may be an artifact caused by a small size of the box of periodicity (an example is the artificial columnar phase in the system of parallel hard spherocylinders [4]). Indeed the observation of the $L$ system showed that the row and 


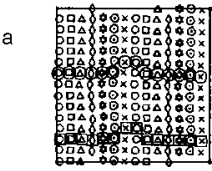

$p=30.0, \quad N=196$

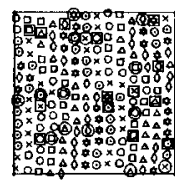

$p=12.0, \quad N=196$

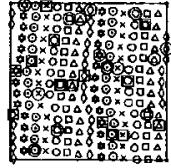

$p=20.0, N=196$

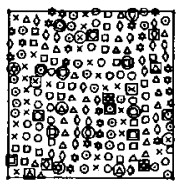

$p=10.0, N=196$

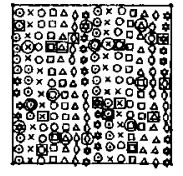

$p=15.0, N=196$

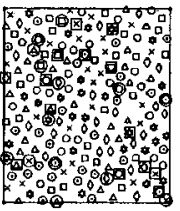

$p=7.0, N=196$

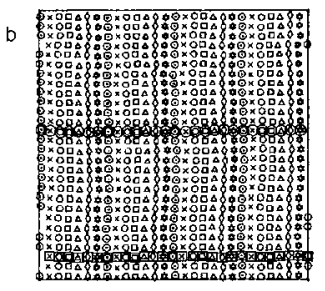

$p=25.0, \quad N=784$

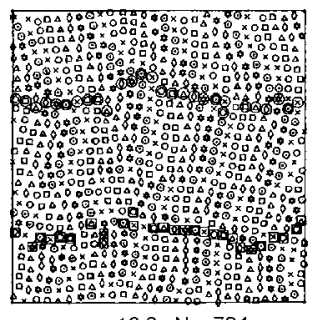

$p=10.0, N=784$

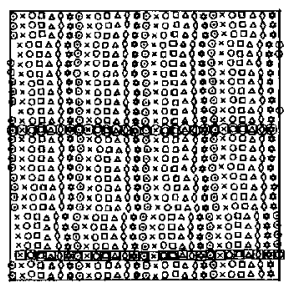

$p=24.0 . \quad N=784$

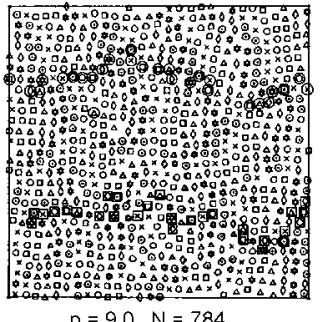

$p=9.0, \quad N=784$

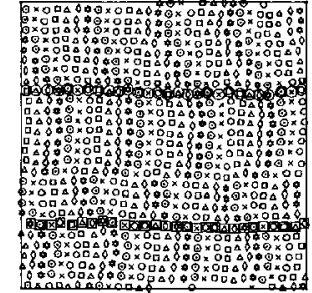

$p=11.0, N=784$

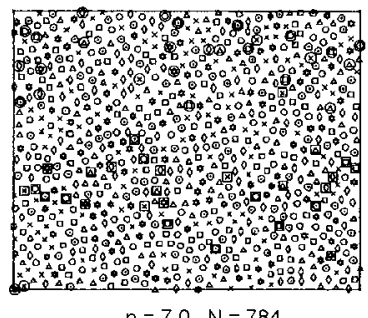

$p=7.0, \quad N=784$

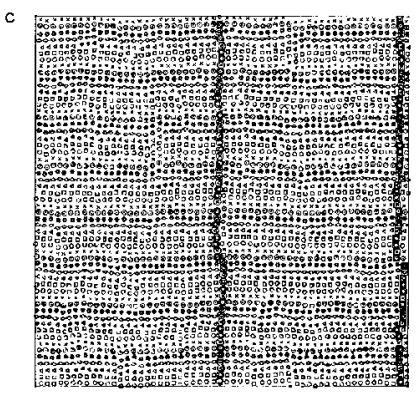

$p=12.0, N=3136$
Fig. 14. Snap-shot configurations of: (a) the $S$ system, (b) the $L$ system, and (c) the $X L$ system. The molecules forming (vertical) columns in the initial perfect structure are represented by the same, small symbols. Larger symbols (circles and squares) represent two (horizontal) rows 
column shifts were much less frequent (Fig. 14b). Moreover, the neighbouring rows and columns were typically 'in phase', forming a square lattice (at least locally). Frequency of the row sliding was even less frequent in the $X L$ system (Fig. 12c); the structure observed there is simply the square lattice with some defects. At high densities the structures observed were, in general, more ordered and closer to the perfect square lattice. Hence, we conclude that the solid phase of the hard squares is the $2 \mathrm{D}$ square crystal.

\section{SUMMARY AND CONCLUSIONS}

The MC simulations performed for $N=196,784$ and 3136 hard squares in a periodic box revealed a tetratic phase (of short range translational order and quasi-long-range bond-bond and molecular orientational order) between the isotropic fluid and a 2D square crystal. Neither a 2D analogue of a 3D smectic or columnar phase nor a 2D plastic crystal has been observed. The transition between the crystalline and tetratic phase appears to be continuous and smooth. The transition between the tetratic phase and isotropic fluid is either continuous (cusp-like) or weekly first order.

If one compares the results concerning the lower and the upper bound of the inter- mediate, tetratic region, it is easy to see that it shrinks with increasing of the system size. Hence, a question arises about stability of this phase in the thermodynamic limit. The present results do not allow us to either prove or disprove stability of the tetratic phase in the thermodynamic limit. The observed strong dependence of the EOS on the system size in the intermediate region seems to be a serious obstacle for solution of this problem. Smart methods based on analysis of the size dependence of the free energy of small systems [39, 40] may be risky in the present case by analogy to the hard disc system. (In the latter case such a method gave a result being in clear contradiction with direct studies performed for large systems [18, 17].) Similar objections can be posed against methods based on the direct evaluation of the free energy in distinct phases. The latter methods are also known to generate artificial results in too small systems (the example is the columnar phase in the hard spherocylinder system; compare the Refs. [4, 41]). Thus, one can expect that use of very long runs and studies of very large systems will be necessary to solve the problem of thermodynamic stability of the tetratic phase in the hard square system.

The simulations prove the existence of strong coupling between the molecular orientations and intermolecular bonds. This implies that both the molecules and bonds have to show the same ordering in the system.

It is worth to add that a simple mean field approximation, smoothed free volume, reproduces the MC results concerning the EOS and the specific heat surprisingly accurate.

It may be interesting to perform analogous studies for the system of hard triangles. If the tetratic phase of the hard squares is induced by the molecular anisotropy, as it is suggested by the model discussed by Kleinert and Janke [33, 34], then the triangle system (as consisted of 
more anisotropic particles) should exhibit a hexatic phase. Otherwise, e.g. if the existence of the tetratic phase in the system of squares is related to the 4-fold symmetry then the system of triangles may behave in a standard way, i.e. form only two phases.

\section{Acknowledgements}

One of us (K. W. W.) is grateful to Drs. A. Brańka, M. Glaser and B. Mulder for helpful conversations, and to people from the FOM - Institute for Atomic and Molecular Physics for hospitality in Amsterdam. The work of the Institute of Molecular Physics of the Polish Academy of Sciences, being a part of the research program of the IFM PAN, was supported by the Commission of the European Communities in Action for Cooperation in Sciences and Technology with Central and Eastern European Countries (No. ERB3510PL920297). Part of the work was performed using computer equipment financed by the Foundation for the Polish Science (PONT). The work of FOM is a part of the research program of FOM and was supported by the Netherlands Organization for Scientific Research (NWO).

\section{References}

[1] B. J. Alder and W. G. Hoover, in Physics of Simple Liquids, Ed. H. N. V. Temperley, J. S. Rowlinson, and G. S. Rushbrooke, (North-Holland, 1968).

[2] J. Vieillard-Baron, J. Chem. Phys. 56, 4729 (1972).

[3] D. Frenkel and B. Mulder, Molec. Phvs. 55, 1171 (1985).

[4] A. Stroobants, H. N. W. Lekkerkerker, and D. Frenkel, Phvs. Rev. Lett. 57, 1452 (1987); Phvs. Rev. A 36, 2929(1987).

[5] K. W. Wojciechowski, A. C. Brańka, and M. Parrinello, Molec. Phvs. 53, 1541 (1984).

[6] A. C. Brańka and K. W. Wojciechowski, Molec. Phys. 72, 941 (1991); ibid. 78, 1513 (1993).

[7] D. Frenkel, in: Liquids, Freezing and Glass Transition, Les Houches LI, Eds. J. P. Hansen, D. Levesque and J. Zinn-Justin, Elsevier (1991).

[8] J. A. C. Veerman and D. Frenkel, Phys. Rev. A 45, 5632 (1992).

[9] K. W. Wojciechowski, D. Frenkel, and A. C. Brańka, Phvs. Rev. Lett. 66, 3168 (1991); Phvsica A 196, 519-545 (1993).

[10] K. W. Wojciechowski, Phys. Rev. B 46, 26 (1992).

[11] L. Onsager, Phys. Rev. 62, 558 (1942); L. Onsager, Ann. NY Acad. Sci. 51, 3441 (1949).

[12] K. W. Wojciechowski and J. Kłos, Nematic phase of cubic symmetiy: Solution of the Frenkel $3 D$-cross model, unpublished.

[13] R. Schneider, Convex bodies: The Brunn-Minkowski Theoiy Vol. 44 of Encyclopedia of Mathematics and its Applications, Ed. G.-C. Rota (Cambridge, 1993).

[14] T. Boublik, Mol. Phys. 29, 421 (1975).

[15] G. Tarjus, P. Viot, S. M. Ricci, and J. Talbot, Mol. Phys. 73, 773 (1991).

[16] K. W. Wojciechowski and D. Frenkel, On the phase diagram of two-dimensional hard rectangle system, unpublished.

[17] J. A. Zollweg and G. V. Chester, Phys. Rev B 46, 11186 (1992).

[18] J. Lee and K. J. Strandburg, Phvs. Rev. B 17, 11190 (1992).

[19] R. E. Peierls, Helv. Phys. Acta 7, 81 (1934).

[20] L. D. Landau and E. M. Lifshitz, Statistical Physics, Part I (Pergamon, Oxford, 1980).

[21] N. D. Mermin, Phys. Rev. A 176, 250 (1968).

[22] The Mermin's proof does not concern the case of the hard body systems. For hard discs the loga rithmic divergence has been demonstrated, in: D. A. Young, B. J. Alder, J. Chem Phys. 60, 1254 (1974).

[23] J. M. Kosterlitz and D. J. Thouless, J. Phvs. C 6, 1181 (1973); Prog. Low Temp. Phvs. B 7, 371 (1978).

[24] J. A. Barker and D. Henderson, Rev. Mod. Phvs. 48, 587 (1976).

[25] F. F. Abraham, Phvs. Rep. 80, 339 (1981).

[26] K. J. Strandburg, Rev. Mod. Phvs. 60, 161 (1988). 
[27] M. A. Glaser and N. A. Clark, in Advances in Chemical Physics, Volume LXXXIII, Ed. I. Prigogine and S. A. Rice (Wilev, 1993).

[28] C. Udink and J. van der Elsken, Phvs. Rev. B 35, 279 (1987).

[29] C. Udink and D. Frenkel, Phys. Rev. B 35, 6933 (1987).

[30] D. R. Nelson and B. I. Halperin, Phvs. Rev. B 19, 2457 (1979).

[31] A. P. Young, Phys. Rev. B 19, 1855 (1979).

[32] R. Pindak et ai, Phys. Rev. Lett. 46, 1135 (1981); S. B. Dierker et al, Phys. Rev. Lett. 56, 1819 (1986); J. D. Brock et al., Phvs. Rev. Lett. 57, 98 (1986); A. Aharonv et al, Phvs. Rev. Lett. 46, 1012 (1986).

[33] H. Kleinert, Phys. Lett. A 130, 443 (1988).

[34] W. Janke and H. Kleinert, Phvs. Rev. Lett. 61, 2344 (1988).

[35] D. R. Nelson and B. I. Halperin, Phys. Rev. B 21, 5312 (1980).

[36] M. J. P. Gingras, P. C. W. Holdsworth, and B. Bergersen, Phys. Rev. A, 6786 (1990); see also the references therein.

[37] J. G. Kirkwood, J. Chem. Phys. 18, 380 (1950).

[38] K. W. Wojciechowski, On the free volume approximation for some anisotropic hard bodies, unpublished.

[39] A. M. Ferrenberg and R. H. Swendsen, Phys. Rev. Lett. 61, 2635 (1988); ibid. 63, 1195 (1989).

[40] J. Lee and J. M. Kosterlitz, Phys. Rev. Lett. 65, 137 (1990).

[41] J. A. C. Veerman and D. Frenkel, Phys. Rev. A 41, 3237 (1990). 